\title{
The manipulation of midsole properties to alter impact characteristics in walking
}

\author{
Carina Price $^{1}$, Glen Cooper ${ }^{2}$, and Richard Jones ${ }^{1}$ \\ ${ }^{1}$ Centre for Health Sciences Research, University of Salford, Salford, UK, ${ }^{2}$ School of Engineering, Manchester \\ Metropolitan University, Manchester, UK
}

\section{Footwear Science}

\section{Abstract}

The midsole of footwear can provide an opportunity to attenuate the impact at the footground interface. The present study was undertaken to quantify impact in walking in different footwear midsoles, comparing footwear thickness and hardness variations. Methods: Footbed thickness $(28-41 \mathrm{~mm})$ and hardness $(30-55$ Shore A) were varied independently in 7 flip-flops. Thirteen subjects walked in the footwear variations on a level walkway in the gait laboratory as lower limb kinematics, vertical ground reaction force and peak positive axial tibial acceleration were quantified. Peak magnitude and time of the acceleration were quantified and the heel-strike transient was characterised for comparison between conditions with a repeated-measures ANOVA. Thickness and hardness variations were also compared using a drop-test protocol to replicate walking. Results: Lower limb joint angles did not vary at heel-strike, however, a faster vertical heel-velocity was recorded in the softer midsoles (e.g. 55 Shore $A=-0.294 \pm 0.055,30$ Shore $A=-0.328 \pm 0.052, p<.001)$. Varying the hardness of the midsoles also significantly altered tibial acceleration and force variables, however limited significant differences existed between the thickness variations in walking. Increasing the hardness of the heel section of the footwear increased the peak positive axial tibial acceleration values, for example increasing Shore A from 30 to 40 resulted in a $35 \%$ increase in this variable. Concurrently, the occurrence of heel-strike transients increased from 5.8\% in the 30 Shore A condition to $22.5 \%, 46.7 \%$ and $71.7 \%$ of all trials in the 40,47 and 55 Shore A conditions respectively. The drop-test protocol replicated the differences evident in the walking protocol despite magnitudes being elevated. Conclusion: Modifying midsole properties of flip-flop footwear, particularly hardness, alters the gait kinematics and the shock experienced by the wearer in walking. This may pose benefits in terms of comfort and reduction in loading to the lower limb, however the influence on foot motion at initial contact and footwear longevity should be further quantified.

Key words: shock, footwear, heel-strike transient, accelerometer, material properties. 


\section{Introduction}

Lower limb musculo-skeletal loading in gait begins with the transmission of stress waves at heel-strike. Part of this loading process produces a heel-strike transient (HST), which has been linked to degenerative changes to tissue (Radin et al., 1991), clinical symptoms (Voloshin and Wosk, 1982) and comfort in walking (Whittle et al., 1994). The midsole of a shoe provides an opportunity to apply a visco-elastic material between the foot-ground interface to reduce the energy transferred at heel contact and the transient (Pratt et al., 1986; Whittle, 1999). Choices of material (including hardness) and shape (including thickness) are constrained by the design specification of the footwear. Design specification restrictions include purpose/activity type, the target market, manufacturing considerations and cost. For decades, athletic footwear companies have manipulated midsole-heel properties in order to assess the effect of hardness and thickness of Ethylene Vinyl Acetate (EVA) constructions in footbeds to provide an effective combination for the comfort and protection of the running consumer (Frederick et al., 1984; Hamill et al., 2011; Milani et al., 1997).

Research has been undertaken to quantify changes in midsole hardness and the effect on variables quantify impact in running and mechanical protocols which replicate running (Frederick et al., 1984; Nigg et al., 1987). Researchers report increased positive peak axial tibial acceleration values in impact assemblies and maximum loading rate of the impact peak in running with increased hardness of footwear (DeWit et al., 1995; Sterzing et al., 2013). Other authors identify that there are no differences in the magnitude of the impact peak of the vertical ground reaction force or the maximum loading rate in running with alterations of hardness, which they attribute to adaptations to eversion at initial contact (Nigg et al., 1987). Similarly, increasing the thickness of the heel section of a running shoe has been demonstrated to reduce peak positive axial tibial acceleration and maximum loading rate of 
the vertical ground reaction force in human (Heidenfelder et al., 2010; TenBroek et al., 2013) and mechanical (Frederick et al., 1984) protocols. It is therefore apparent that manipulating midsole thickness and hardness can alter impact characteristics in both human test protocols and mechanical protocols which aim to replicate running. These alterations can include potentially positive outcomes for wearers such as reduced lower limb loading (Hamill et al., 2011; TenBroek et al., 2013) and reduced sensations of impact severity (Lake and Lafortune 1998).

Athletic footwear has provided the basis for most recent work into footbed construction, with walking studies limited to orthotic interventions as opposed to modifications to footwear itself (Healy et al., 2010; Pratt et al., 1986). Thus, there is not currently an extensive investigation of the influence of different footbed characteristics on impact characteristics in walking. The impact with the floor in running is defined by a heel velocity of approximately $1 \mathrm{~m} \cdot \mathrm{s}^{-2}$ and effective mass of $8.5 \mathrm{~kg}$ (Misevich and Cavanagh, 1984). In walking the comparable variables are $0.17-0.36{\mathrm{~m} \cdot \mathrm{s}^{-2}}^{-2}$ and $1.6-17.0 \mathrm{~kg}$ identifying different kinematics and loading magnitudes and rates of the lower limb, as a result of both gait and footwear style (Jørgensen and Bojsen-Møller, 1989; Jefferson et al., 1990; Price et al., 2014). Consistently, recent data has identified that the mechanical protocol utilised to quantify the shock absorption properties in athletic footwear over-estimates the peak acceleration and HST magnitude and underestimates the timing of these variables in walking footwear (Price et al., 2014). The importance of this discrepancy is enhanced by the shock absorption characteristics of viscoelastic materials being rate dependent (Whittle, 1999). Thus, gait specific testing is required to establish the suitability of walking footwear in protocols specific to their 'real-world' wear. A gait specific test has been designed to be implemented within footwear companies to quantify alterations in shock absorption with 
systematic modifications in footwear properties, such as EVA construction (Price et al., 2014).

Despite the focus of research literature on running, walking is a more relevant activity to the general and clinical populations. The different design of running footwear compared to some styles of walking footwear with lace-up, covered uppers, reinforced counters and rubber midsoles and outsoles is evident. In addition to the aforementioned loading characteristics, these factors combine to indicate that findings and recommendations from running studies cannot be inferred to research and development of different styles of walking footwear. In particular, flip-flops represent a footwear style which is anecdotally criticised for not protecting the wearer's lower limb and yet can easily be modified to accommodate a thicker midsole. The increased vertical heel velocity toward the floor in this footwear style also means that the importance of appropriate shock absorption properties may be increased in comparison to other walking footwear styles (Price et al., 2014). Modifying footwear based on walking gait may enable increased comfort and reduced clinical symptoms in these populations (Voloshin and Wosk, 1982; Whittle et al., 1994). Recently the health footwear market has developed and expanded, which can feasibly accommodate changes in footbed thickness and materials in designs as long as benefits can be justified to consumers. The study of these thickness and hardness alterations is therefore warranted with test protocols that include walking protocols to infer footwear design for specific footwear styles.

The primary aim of the study was to quantify the effects of differing midsole hardness and thickness on impact variables in flip-flop footwear tests during walking and in a mechanical protocol to replicate walking in flip-flops. It is expected that increasing footbed thickness and decreasing hardness would reduce peak acceleration and forces in walking protocols due to the provision of a longer time to apply force and a more viscoelastic material to absorb more energy from the touchdown (Whittle, 1999). 


\section{Methods}

Ethical approval for the study was achieved through the University ethics committee; volunteers were recruited from the staff and student populations.

\subsection{Footwear Tested}

Seven footwear conditions were tested with varying midsole depths and hardness in a flipflop upper (Table 1) using mechanical and human methodologies. The shoes were varied only in the heel characteristics, all other shoe features were consistent (upper/pitch/outsole/profile etc) and were prototype versions of the FitFlop ${ }^{\mathrm{TM}}$ walkstar. Due to constraints in manufacture, the upper differed between the hardness and thickness shoes, but was consistent within them. The thickness variations had a toe-post upper, the hardness a sandal upper with no back-strap.

Table 1. Footwear characteristics for the seven footwear conditions tested in the study, all of which had a sandal upper and an EVA construction.

\begin{tabular}{lccc}
\hline \multicolumn{1}{c}{ Condition } & $\begin{array}{c}\text { Heel Depth } \\
(\mathbf{m m})\end{array}$ & $\begin{array}{c}\text { Heel Hardness } \\
\text { (Shore A) }\end{array}$ \\
\hline Thickness & $\mathrm{T} 41$ & 41 & 40 \\
Variations & $\mathrm{T} 35$ & 45 & \\
& $\mathrm{~T} 28$ & 28 & 55 \\
& & 41 & 47 \\
Hardness & H55 & 41 & 40 \\
Variations & H47 & 41 & 30 \\
& H40 & 41 & \\
\hline
\end{tabular}

Where Shore A hardness was measured with a durometer and a bespoke device, which is utilised for quality control and implements a larger base to contact the test specimen. 


\subsection{Protocol}

Thirteen healthy subjects ( 2 males, 11 females, $27.5 \pm 8.8$ years, $62.0 \pm 10.3 \mathrm{~kg}, 1.65 \pm 0.05$ metres, mean \pm 1 S.D) with shoe size U.K. 6 gave their consent and participated in the study. Subjects reported no lower limb injury in order to take part in the study and were instrumented with a lower limb marker setup for 3-D motion capture and one uni-axial accelerometer.

A 10 camera Qualisys Pro-Reflex system (Qualisys, Sävebalden, Sweden) was used to track 3D motion at $240 \mathrm{~Hz}$. Spherical retro-reflective markers and clusters were positioned to define the lower limbs in accordance with the CAST technique (Cappozzo et al., 1995). The foot was defined with markers on the posterior calcaneus and the dorsal aspects of the 1st, 2nd and 5th metatarsal heads. The shank was defined with anatomical markers on the medial and lateral malleoli and the medial and lateral knee, with a rigid plate of four tracking markers on the anterior tibia. The accelerometer was mounted on the right anterior-medial tibia above the medial malleolus on a small piece of light flexible plastic. It was positioned 5-10 $\mathrm{cm}$ above the malleolus, on an area with least adipose tissue, oriented with the tibia axis. The accelerometer was affixed with double-sided tape and an elasticated bandage secured it tightly without causing discomfort. The accelerometer was sampled alongside 2 force plates (AMTI, Advanced Mechanical Technology, Watertown, USA) at $2400 \mathrm{~Hz}$ collecting ground reaction force data for two consecutive right heel-strikes. Subjects performed 5 trials in each condition in a randomised order following a familiarisation period of 4 practice walks, data from the right leg only was utilised. Ten data-sets for each footwear condition were analysed. Participants walked at a self-selected velocity for the first condition which was then monitored with timing gates to ensure consistent walking speeds within a range of $\pm 5 \%$, trials outside this boundary were re-captured. 
The drop-test methodology has previously been described and utilised a protocol which replicated the energy of the shoe-ground impact in walking (Price et al., 2014). The footwear conditions were impacted with a mass of $17 \mathrm{~kg}$ from a drop height of $5 \mathrm{~mm}$ to replicate the impact characteristics evident in this style of footwear during walking (Price et al., 2014). This compares to the $8.5 \mathrm{~kg}$ and $50 \mathrm{~mm}$ utilised in the standard ASTM protocol F1614 (Procedure A).

\subsection{Data Processing}

Data was processed and analysed using Visual 3D (C-Motion, Inc., Rockville, MD, USA), defining the right limb and pelvis as 4 rigid segments. 3D motion $(10 \mathrm{~Hz})$ and accelerometer $(100 \mathrm{~Hz})$ data was filtered using low-pass Butterworth filters. Ground reaction force data was not filtered due to findings from Gillespie and Dickey (2003). Force plate contact was defined as the first frame in which the vertical ground reaction force (vGRF) exceeded 4N. Joint angles at heel-strike for the sagittal plane at the ankle, knee and hip were computed for the concurrent frame for which force plate contact was defined. Heel-marker vertical velocities at heel-strike were calculated using the mean value from $8 \mathrm{~ms}$ leading up to heel-

strike, which is within ranges found to be reliable in previous research (Karst et al., 1999). Heel-strike transient (HST) of the vertical GRF was defined as a local maximum point between the $4 \mathrm{~N}$ vGRF threshold and the first vGRF peak. This was computed using Newton's difference quotient with a central derivative approximation, to identify zero gradient of the vGRF. The magnitude of vGRF at the HST and time of this variable were quantified. Maximum instantaneous loading rate of the vGRF was computed for all trials from the difference quotient. Magnitude and timing of peak positive axial tibial acceleration was also calculated and used to compute the rate to peak positive axial tibial acceleration. Temporal-spatial data (including step length and stance time) was calculated automatically 
and output for comparison.

Statistical comparison was undertaken between hardness and thickness variations in SPSS (SPSS Inc., Chicago), using ANOVA with Bonferonni correction for multiple comparisons ( $p$ value $<0.05$ ). The number of HST in each condition was compared statistically prior to conversion to percentages of total trials for presentation and HST data was not compared statistically due to inconsistent and small $\mathrm{N}$ numbers.

\section{Results}

The comparison of kinematic variables in walking identified no significant differences between thickness or hardness variations in lower limb sagittal plane joint angles at heelstrike, or temporal-spatial characteristics (Table 2). Vertical heel velocities at heel-strike differed between the hardness conditions, decreasing as the hardness of the footwear heel section increased (Table 2). No differences were evident in this variable in the thickness variations. 
Table 2 Kinematic data from walking in different hardness and thickness variations: Joint angles for the ankle, knee and hip and vertical heel velocity at heel-stike (mean \pm standard deviation).. Statistically significant (ANOVA $p<0.05)$ results are presented.

\begin{tabular}{|c|c|c|c|c|c|c|c|c|c|}
\hline \multirow{3}{*}{ Variables } & \multicolumn{7}{|c|}{ Footwear Condition } & \multirow{2}{*}{\multicolumn{2}{|c|}{ Significant $p$ values }} \\
\hline & \multicolumn{4}{|c|}{ Thickness } & \multicolumn{3}{|c|}{ Hardness } & & \\
\hline & T41 & T35 & T28 & H55 & H47 & H40 & H30 & Thickness & Hardness \\
\hline Ankle $\left(^{\circ}\right)$ & $4.7 \pm 4.1$ & $4.0 \pm 3.8$ & $4.3 \pm 3.1$ & $4.0 \pm 3.7$ & $4.2 \pm 3.9$ & $3.8 \pm 3.6$ & $4.0 \pm 4.1$ & - & - \\
\hline Knee $\left(^{\circ}\right)$ & $-0.5 \pm 4.0$ & $-0.5 \pm 3.8$ & $-1.3 \pm 3.5$ & $-0.4 \pm 5.4$ & $-0.7 \pm 4.9$ & $0.1 \pm 5.7$ & $-1.3 \pm 4.2$ & - & - \\
\hline $\operatorname{Hip}\left(^{\circ}\right)$ & $25.7 \pm 6.5$ & $25.7 \pm 6.4$ & $25.4 \pm 7.0$ & $23.5 \pm 6.9$ & $24.1 \pm 6.3$ & $23.8 \pm 6.2$ & $24.7 \pm 7.4$ & - & - \\
\hline $\begin{array}{l}\text { Vertical Heel Velocity } \\
\left(\mathrm{m} \cdot \mathrm{s}^{-1}\right)\end{array}$ & $-0.358 \pm 0.055$ & $-0.376 \pm 0.065$ & $-0.378 \pm 0.057$ & $-0.294 \pm 0.055$ & $-0.292 \pm 0.055$ & $-0.315 \pm 0.049$ & $-0.328 \pm 0.052$ & - & $\begin{array}{c}\mathrm{H} 55<\mathrm{H} 40 \mathrm{p}=.003 \\
\mathrm{H} 55<\mathrm{H} 30 \mathrm{p}<.001 \\
\mathrm{H} 47<\mathrm{H} 30 \mathrm{p}<.001 \\
\mathrm{H} 40<\mathrm{H} 30 \mathrm{p}=.009 \\
\mathrm{H} 47<\mathrm{H} 40 \mathrm{p}=.027\end{array}$ \\
\hline Step Length (m) & $0.680 \pm 0.030$ & $0.679 \pm 0.032$ & $0.678 \pm 0.029$ & $0.679 \pm 0.023$ & $0.687 \pm 0.029$ & $0.679 \pm 0.023$ & $0.680 \pm 0.026$ & - & - \\
\hline
\end{tabular}

Where T41= $41 \mathrm{~mm}, \mathrm{~T} 34=34 \mathrm{~mm}$ and T28 = $28 \mathrm{~mm}$ of heel depth and H55= 55 Shore A, H47 = $47 \mathrm{Shore} \mathrm{A}, \mathrm{H} 40=40 \mathrm{Shore} \mathrm{A}$ and H30 = 30

Shore A hardness in the heel section 


\subsection{Thickness}

Analysis of HST identified the feature occurred in $46.9 \%$ of the thickness variation trials collected and did not significantly vary between conditions (Table 3). Analysis of the HST magnitude demonstrated an increase in magnitude of HST with decreasing footbed thickness (Table 3). Consistent with the force variable, peak positive axial tibial accelerations displayed a trend to increase with decreasing thickness. However, no significant differences were evident in human acceleration variables between thickness conditions, despite the T28 condition producing a $10.3 \%$ increase in peak positive axial tibial acceleration compared to T41. The only significant difference between the thickness conditions in the human data was that loading rate in the thinnest condition (T28) was higher than in T35 (Table 3). The droptest protocol identified significantly lower peak acceleration and force in the thinnest condition (Table 3).

\subsection{Hardness}

Analysis of walking in the hardness conditions, demonstrated the HST feature occurred in $37.5 \%$ of the trials (Table 4). The H30 condition (the softest EVA tested) produced HST in a total of 8 trials from 4 participants, in contrast walking in the $\mathrm{H} 55$ condition resulted in a HST in $71.7 \%$ of all trials and only 3 participants did not demonstrate HST in this condition. The magnitude of the HST increased and the feature occurred a shorter duration from heel contact following alterations in footbed hardness, although these variables were not explored statistically (Table 4). The maximum instantaneous loading rate also reflected this trend and decreased with reduced hardness. Although, despite a $5.7 \mathrm{kN} \cdot \mathrm{s}^{-1}$ change, this variable did not significantly differ between the $\mathrm{H} 40$ and $\mathrm{H} 30$ conditions. Peak positive axial tibial accelerations increased as hardness of the footbed increased. The magnitude of peak positive 
axial tibial acceleration reduced by $12.8 \%$ in $\mathrm{H} 55$ compared to $\mathrm{H} 47$ and by $28.1 \%$ and $46.8 \%$ respectively in $\mathrm{H} 55$ in comparison to $\mathrm{H} 40$ and $\mathrm{H} 30$. The time of peak positive axial tibial acceleration was later with softer EVA, therefore rate to peak positive axial tibial acceleration also significantly increased as hardness decreased (Table 4). The drop-test protocol demonstrated significant decreases in both peak force and acceleration with reducing hardness until $\mathrm{H} 30$, for which magnitudes increased compared to $\mathrm{H} 40$.

Table 3 Heel-strike transient and peak positive axial tibial acceleration variables for thickness variations.

\begin{tabular}{|c|c|c|c|c|c|}
\hline \multirow{2}{*}{ Variables } & \multicolumn{4}{|c|}{ Thickness Condition } & \multirow[b]{2}{*}{ Significant $p$ values } \\
\hline & & T41 & T35 & $\mathbf{T} 28$ & \\
\hline \multirow{8}{*}{$\begin{array}{l}\text { Human } \\
\text { Testing }\end{array}$} & $\begin{array}{l}\text { Percentage of all } \\
\text { trials with HST }(\%)\end{array}$ & 43.3 & 51.7 & 45.8 & - \\
\hline & HST magnitude $(\mathrm{N})$ & $305.8 \pm 113.3$ & $332.4 \pm 155.8$ & $366.7 \pm 117.4$ & NA \\
\hline & HST time (ms) & $31.9 \pm .04$ & $32.5 \pm 0.4$ & $32.3 \pm 0.6$ & NA \\
\hline & $\begin{array}{l}\text { Maximum } \\
\text { Instantaneous } \\
\text { Loading Rate }\left(\mathrm{kN} \cdot \mathrm{s}^{-1}\right)\end{array}$ & $22.0 \pm 7.0$ & $21.8 \pm 7.1$ & $23.9 \pm 8.6$ & $\mathrm{~T} 28>\mathrm{T} 35 \mathrm{p}=.038$ \\
\hline & Peak Positive Axial & & & & \\
\hline & $\begin{array}{l}\text { Tibial Acceleration } \\
\left(\mathrm{m} \cdot \mathrm{s}^{-2}\right)\end{array}$ & $17.4 \pm 8.4$ & $18.1 \pm 8.9$ & $21.0 \pm 10.6$ & - \\
\hline & $\begin{array}{l}\text { Time of Peak } \\
\text { Positive Axial Tibial } \\
\text { Acceleration (ms) }\end{array}$ & $25.4 \pm 6.9$ & $24.0 \pm 5.8$ & $25.4 \pm 10.7$ & - \\
\hline & $\begin{array}{l}\text { Rate to Peak Positive } \\
\text { Axial Tibial } \\
\text { Acceleration }\left(\mathrm{m} \cdot \mathrm{s}^{-3}\right)\end{array}$ & $692.2 \pm 336.3$ & $745.0 \pm 363.3$ & $858.8 \pm 500.2$ & - \\
\hline Mechanical & $\begin{array}{l}\text { Peak Acceleration } \\
\left(\mathrm{m} \cdot \mathrm{s}^{-2}\right)\end{array}$ & $32.9 \pm 2.6$ & $33.7 \pm 1.5$ & $34.9 \pm 0.7$ & - \\
\hline Impact tester & $\begin{array}{l}\text { Peak Force } \\
(\mathrm{N})\end{array}$ & $669.7 \pm 29.3$ & $700.1 \pm 20.5$ & $687.8 \pm 8.3$ & $\mathrm{~T} 41<\mathrm{T} 35 \mathrm{p}=.037$ \\
\hline
\end{tabular}

Where T41 $=41 \mathrm{~mm}, \mathrm{~T} 34=34 \mathrm{~mm}$ and T28 $=28 \mathrm{~mm}$ of heel depth. Data is presented as mean \pm standard deviation. Statistically significant (ANOVA $p<0.05$ ) $\mathrm{p}$ values are presented. HST magnitude and time are presented for the trials that included a HST only. 
Table 4. Kinematic and kinetic impact variables for heel-strike trials accelerometer in the hardness variations (mean \pm standard deviation). Statistically significant (ANOVA $p<0.05$ ) results are presented.

\section{Hardness Condition}

Variables

H55

H47

H40

H30

Significant $p$

values

\begin{tabular}{|c|c|c|c|c|c|}
\hline $\begin{array}{l}\text { Percentage of all trials with } \\
\text { HST }(\%)\end{array}$ & 44.6 & 15.4 & 2.3 & 0 & $\begin{array}{l}\mathrm{H} 55>\mathrm{H} 47 \mathrm{p}=.007 \\
\mathrm{H} 55>\mathrm{H} 40 \mathrm{p}=.017 \\
\mathrm{H} 55>\mathrm{H} 30 \mathrm{p}=.008\end{array}$ \\
\hline $\begin{array}{l}\text { Average HST magnitude } \\
\text { (N) }\end{array}$ & $371.1 \pm 142.3$ & $290.7 \pm 29.8$ & $344.7 \pm 81.8$ & - & NA \\
\hline Time HST (milliseconds) & $28.5 \pm 0.4$ & $31.5 \pm 0.5$ & $39.8 \pm 1.2$ & - & NA \\
\hline Loading rate to HST $\left(\mathrm{kN} . \mathrm{s}^{-1}\right)$ & $13.1 \pm 3.0$ & $9.8 \pm 0.9$ & $8.1 \pm 0.1$ & - & NA \\
\hline $\begin{array}{l}\text { Peak Tibial Acceleration } \\
\left(\mathrm{m} . \mathrm{s}^{-2}\right)\end{array}$ & $23.5 \pm 9.2$ & $20.5 \pm 7.9$ & $16.9 \pm 4.5$ & $12.5 \pm 3.2$ & $\begin{array}{l}\mathrm{H} 55>\mathrm{H} 40 \mathrm{p}=.008 \\
\mathrm{H} 55>\mathrm{H} 30 \mathrm{p}=.001 \\
\mathrm{H} 47>\mathrm{H} 40 \mathrm{p}=.039 \\
\mathrm{H} 47>\mathrm{H} 30 \mathrm{p}=.003\end{array}$ \\
\hline $\begin{array}{l}\text { Time of Peak Tibial } \\
\text { Acceleration (milliseconds) }\end{array}$ & $19.4 \pm 5.3$ & $21.0 \pm 7.7$ & $24.4 \pm 8.3$ & $26.7 \pm 9.2$ & $\begin{array}{l}\mathrm{H} 55<\mathrm{H} 40 \mathrm{p}=.046 \\
\mathrm{H} 47<\mathrm{H} 40 \mathrm{p}=.036\end{array}$ \\
\hline $\begin{array}{l}\text { Rate to Peak Tibial } \\
\text { Acceleration }\left(\mathrm{m}^{-\mathrm{s}^{-3}}\right)\end{array}$ & $1165.2 \pm 436.4$ & $961.8 \pm 378.1$ & $697.0 \pm 275.9$ & $495.9 \pm 198.9$ & $\begin{array}{l}\mathrm{H} 55>\mathrm{H} 40 \mathrm{p}=.000 \\
\mathrm{H} 55>\mathrm{H} 30 \mathrm{p}=.000 \\
\mathrm{H} 47>\mathrm{H} 40 \mathrm{p}=.000 \\
\mathrm{H} 47>\mathrm{H} 30 \mathrm{p}=.000 \\
\mathrm{H} 40>\mathrm{H} 30 \mathrm{p}=.002\end{array}$ \\
\hline
\end{tabular}

Where H55= 55 Shore A, H47 = 47 Shore A, H40 = 40 Shore A and H30 = 30 Shore A

hardness in the heel section.

\section{Discussion}

The aim of the study was to quantify the effects of differing midsole hardness and thickness on shock absorption variables in flip-flop footwear. Therefore other aspects of the footwear including outsole shape and upper characteristics were not varied. The study identified significant differences between thickness and hardness midsole variations when being assessed for shock absorption using both human and mechanical walking protocols. 
The temporal-spatial and kinematic data comparison identified limited significant differences within the thickness and hardness variations. The hardness variations recorded a lower vertical heel velocity towards the floor than the thickness variations (e.g. H55 $0.294 \pm 0.055$ v T35 $-0.376 \pm 0.065)$, likely due to the differing uppers (Price et al., 2014). Also within the hardness variations the participants' heel velocity was systematically faster in the softest conditions after a hardness of 47 Shore A. These results demonstrate to a footwear designer or technologist that, within the hardness and thicknesses ranges tested in this study and population in this research, modifying hardness alters heel contact velocity, but modifying thickness does not. This means that if a footwear designer is to change the footbed hardness of flip-flop footwear they must consider how this influences the velocity at heelstrike when considering aspects such as shock absorption, comfort and product longevity. Despite not influencing vertical heel-velocity at touchdown in this study, it is probable that modifications to footbed thickness may alter kinematics in terms of swing characteristics within footwear due to the demands of toe-clearance (Menant et al., 2009). In other footwear styles and gait modalities, Kersting and Brüggemann (2006) identified minimal and nonsignificant variations in the touchdown velocity of the malleoulus in trainers with differing midsole hardness (45-61 Shore C) in running, consistent with Nigg et al. (1987) in running shoes of 25-45 Shore A. Despite the changes in heel velocity apparent in the present research, no significant differences were evident in lower limb sagittal plane joint angles at heel-strike within the hardness (e.g. H55 v H47) or thickness (e.g. T41 v T28) variations when walking in flip-flops. Previous research in running has identified significant kinematic adaptations to knee flexion to mediate the stiffness of the limb and reduce impact energy, however in running limiting the maximum forces due to impact in the system may be more essential than in walking (impact forces may not exceed these limits in walking gait). 


\subsection{Thickness}

It was hypothesised that decreasing the thickness of the footbed would increase the occurrence and magnitude of the HST and the magnitude of the peak positive axial tibial acceleration. The HST is caused by the force-time characteristics of the impact as the foot/shoe strikes the ground and is measured by the force plate. A stress wave from this impact travels proximally through the foot and into the limb. The magnitude of the force evident can be reduced by viscoelastic footbed material. The dissipation will be proportional to the damping coefficient of the material and the amount of material it travels through, hence thicker midsoles will reduce the magnitude of the HST. Despite differing gait and footwear styles, this is consistent with previous research in running footwear where increased peak acceleration values and a trend for increased force loading rate were evident in thicker footbeds (Hamill et al., 2011; TenBroek et al., 2013).

Both HST and peak positive axial tibial acceleration in the current research reduced with increasing midsole thickness, however differences were not statistically significant in the human flip-flop walking data. . The drop-test protocol also largely failed to differentiate between the thickness variations tested. Maximum instantaneous loading rate of the vGRF was significantly higher in T28 than T35. These results suggest that potentially reducing an item of footwear with this construction from 41 to $35 \mathrm{~mm}$ in the heel may not results in any evident reduction in shock absorption properties, however further reductions may be detrimental. It may be apparent that the additional $13 \mathrm{~mm}$ of EVA may be redundant in terms of shock absorption capacity for flip-flop footwear. It is an example as to why other factors such as longevity of the foam at different thicknesses would also need to be considered in design. Thicker foam in a walking shoe may absorb slightly more shock and last longer, however the cost of manufacture and distribution is increased so the specific product requirements should be considered. 
The identification of significant differences between conditions may have been limited by a large range in individual response, which resulted in large deviations about the mean values for the variables (for example standard deviations for peak acceleration were 8.4-10.6 m.s ${ }^{-2}$ and HST transient 113.3-155.8 N). A greater range of thicknesses may have identified further differences and also been more generalisable to the wider walking footwear market as opposed to this specific flip-flop style. Also the thinnest condition $(28 \mathrm{~mm})$ is also relatively thick for an EVA footbed in a walking shoe, but relevant to 'health and well-being' footwear.

\subsection{Hardness}

Consistent with the study hypothesis, the variations in hardness of the footbeds in the current study produced significantly lower peak axial tibial accelerations and reduced loading rates in softer footbeds. Also, the occurrence of HST reduced and the HST occurred later from heel contact in softer footbeds. The reduced occurrence is consistent with a reduced transmission of energy from impact in softer soled footwear. Less viscoelastic footbed materials, due primarily to reduced viscosity, absorb less energy such that recorded force is higher. Meaning that the magnitude of the HST is proportional to the viscoelasticity of the midsole when the thickness of the sole is un-changed. As the behaviour of the viscous component is ratedependent it is essential that the rate and conditions of the loading reflect the intended use of the footwear, therefore data from running tests is not suitable to explain the response of footbeds in walking shoes. Contrasting this expectation, the peak positive axial tibial acceleration did not differ significantly between the two hardest and two softest conditions respectively. Similar to the thickness results, this identifies that footbed modification within certain ranges result in negligible alterations to the loading experienced by the wearer when walking in flip-flops. 
The HST magnitude increased with decreasing hardness, which may be a function of the individual participant response. As the conditions became softer, fewer participants had evident HST which meant that the mean values were more heavily weighted toward participants with more severe HST. Similarly, in running shoes, Nigg et al. (1987) identified no difference in maximum force between hard (45 Shore A) and soft (25 Shore A) conditions. This was attributed to changes in initial eversion patterns. Further analysis of motion data in the current work would be required to determine adjustments are apparent in the present work, however this is beyond the scope of the present comparison. Contrasting the work by Nigg et al. (1987) pertaining to running, in the current study the loading rates however did decrease as hardness decreased, consistent with other previous research in running (DeWit et al., 1995). This supports the suggestion by Hennig (2011) that the force loading rate is the most representative variable when considering the shock absorption properties of footwear in-vivo and particularly due to the aforementioned data analysis process implemented in the current research for HST variables.

The drop-test results reduced progressively with decreasing hardness until the softest condition where the peak acceleration and peak force variables increased to a level consistent with the $\mathrm{H} 47$ condition. This may be an indication of the material bottoming-out in response to the load applied and the rate of loading. As this was not evident in the walking data it is also an indication that, despite the modification, the mechanical testing methodology does not accurately represent the loading evident in these participants.

\subsection{Limitations}

Individual subject variability in the current study may have affected the HST magnitude, as the HST feature is not evident in all subjects for all conditions, so the mean data is influenced by which individual subject recorded a transient in each condition and variability between 
them. In running the first peak in vGRF is a feature apparent in all runners (Cavanagh and Lafortune, 1980) and therefore mean data between conditions includes all test subjects. Limitations are apparent in the present study, particularly the high vertical heel velocity and kinematics in the footwear tested due to the sandal upper means that the results may not be transferrable to all footwear styles and uppers (Lake and Robinson, 2005; Price et al., 2014; Shroyer and Weimar, 2010). The lack of testing of the interaction of material hardness and thickness also limits the application of results as footwear technologists are likely to manipulate thickness and hardness of EVA in combination as opposed to in isolation. Further work to quantify the influence of the thickness and hardness variations on foot motion and durability of footwear in walking is recommended.

\subsection{Conclusions}

The present study highlights that adaptations of footbed properties of daily walking footwear can significantly alter the impact characteristics experienced by the wearer. This study points to softer footbeds offering advantages in shock absorption, however their impact on motion at heel-strike as well as the longevity of softer foams should be considered prior to their recommendation for use in walking footwear manufacture. The differences evident in the thickness of the footbeds identified minimal differences in the shock absorption capability of 28-41 mm thick EVA footbeds in walking. The range of thicknesses employed in this study did not alter gait kinematics at heel-strike, however the alterations in hardness instigated altered heel contact velocity, which has implications for footwear design. Future work should determine the meaning of the magnitude of variables in terms of comfort or injury and potentially a recommended threshold for shock absorption properties in walking footwear. 


\section{Conflict of interest statement}

The primary author of the paper worked on a research project which was funded by FitFlop ltd, supervised by Dr Jones. Dr G. Cooper was not funded by the research project. Work was undertaken with scientific diligence, data was collected, analysed and the paper written with no influence from the funding company.

\section{References}

Cavanagh, P.R., Lafortune, M.A., 1980. Ground reaction forces in distance running. Journal of Biomechanics 13, 397-406.

Cappozzo, A., Catani, F., Croce, U.D., Leardini, A., 1995. Position and orientation in space of bones during movement: anatomical frame definition and determination. Clin Biomech (Bristol, Avon) 10, 171-178.

DeWit, B. De Clercq, D., Lenoir, M. 1995. The effect of varying midsole hardness on impact forces and foot motion during foot contact in running. Journal of Applied Biomechanics 11, 395-406.

Frederick, E., Clarke, T., Hamill, C., 1984. The effect of running shoe design on shock attenuation. In: Sport Shoes and Playing Surfaces. Human Kinetics, Champaign, IL, pp. 190-198.

Hamill, J., Russell, E.M., Gruber, A.H., Miller, R., 2011. Impact characteristics in shod and barefoot running. Footwear Science 3, 33-40.

Healy, A., Dunning, D.N., Chockalingam, N., 2010. Materials used for footwear orthoses: a review. Footwear Sci. 2, 93-110.

Heidenfelder, J., Sterzing, T., Bullmann, M., Milani, T.L., 2010. Heel strike angle and foot angular velocity in the sagittal plane during running in different shoe conditions. $J$ Foot Ankle Res 1, O16-O16.

Hennig, E.M., 2011. Eighteen years of running shoe testing in Germany - a series of biomechanical studies. Footwear Science 3, 71-81. 
Gillespie, K.A., Dickey, J.P., 2003. Determination of the effectiveness of materials in attenuating high frequency shock during gait using filterbank analysis. Clin Biomech (Bristol, Avon) 18, 50-59.

Jefferson, R.J., Collins, J.J., Whittle, M.W., Radin, E.L., O’Connor, J.J., 1990. The Role of the Quadriceps in Controlling Impulsive Forces around Heel Strike. Proceedings of the Institution of Mechanical Engineers, Part H: Journal of Engineering in Medicine $204,21-28$.

Jørgensen, U., Bojsen-Møller, F., 1989. Shock absorbency of factors in the shoe/heel interaction--with special focus on role of the heel pad. Foot Ankle 9, 294-299.

Karst, G.M., Hageman, P.A., Jones, T.F., Bunner, S.H., 1999. Reliability of Foot Trajectory Measures Within and Between Testing Sessions. The Journals of Gerontology Series A: Biological Sciences and Medical Sciences 54, M343 -M347.

Kersting, U.G., Brüggemann, G.P., 2006. Midsole material-related force control during heeltoe running. Research in Sports Medicine, 14(1), 1-17.

Lake, M.J., Lafortune, M.A., 1998. Mechanical inputs related to perception of lower extremity impact loading severity. Med Sci Sports Exerc 30, 136-143.

Lake, M., Robinson, M., 2005. Biomechanics of walking in different shoes: a comparison between overground and treadmill testing protocols, in: 7th Symosium on Footwear Biomechanics. Presented at the Footwear Biomechanics Group, Cleveland, Ohio.

Menant, J.C., Steele, J.R., Menz, H.B., Munro, B.J., Lord, S.R., 2009. Effects of walking surfaces and footwear on temporo-spatial gait parameters in young and older people. Gait \& Posture 29, 392-397.

Milani, T.L., Hennig, E.M., Lafortune, M.A., 1997. Perceptual and biomechanical variables for running in identical shoe constructions with varying midsole hardness. Clin Biomech (Bristol, Avon) 12, 294-300.

Misevich, K., Cavanagh, P., 1984. Material Aspects of Modeling Shoe/Foot Interaction, in: Sport Shoes and Playing Surfaces. Human Kinetics, Champaign, IL, pp. 47-75.

Nigg, B.M., Bahlsen, H.A., Luethi, S.M., Stokes, S., 1987. The influence of running velocity and midsole hardness on external impact forces in heel-toe running. J Biomech 20, 951-959.

Pratt, D.J., Rees, P.H., Rodgers, C., 1986. Technical Note: Assessment of Some Shock Absorbing Insoles. Prosthetics and Orthotics International 10, 43 -45.

Price, C., Cooper, G., Graham-Smith, P., Jones, R., 2014. A mechanical protocol to replicate impact in walking footwear. Gait \& Posture 40, 26-31. 
Radin, E.L., Yang, K.H., Riegger, C., Kish, V.L., O’Connor, J.J., 1991. Relationship between lower limb dynamics and knee joint pain. Journal of Orthopaedic Research 9, 398405.

Shroyer, J.F., Weimar, W.H., 2010. Comparative Analysis of Human Gait While Wearing Thong-Style Flip-flops versus Sneakers. J Am Podiatr Med Assoc 100, 251-257.

Sterzing, T., Schweiger, V., Ding, R., Cheung, J.T.-M., Brauner, T., 2013. Influence of rearfoot and forefoot midsole hardness on biomechanical and perception variables during heel-toe running. Footwear Science 1-9.

TenBroek, T.M., Rodrigues, P., Frederick, E.C., Hamill, J., 2013. Effects of unknown footwear midsole thickness on running kinematics within the initial six minutes of running. Footwear Science 5, 27-37.

Voloshin, A., Wosk, J., 1982. An in vivo study of low back pain and shock absorption in the human locomotor system. J Biomech 15, 21-27.

Whittle, M., Orofino, T., Miller, K., 1994. Technical approach to characterization of perceived comfort of walking surfaces. Gait \& Posture 2, 129-133.

Whittle, M.W., 1999. Generation and attenuation of transient impulsive forces beneath the foot: a review. Gait Posture 10, 264-275. 